\title{
Tomography of Interacting Binary Systems: Algols to Gamma-Ray Binaries
}

\author{
Mercedes T. Richards \\ Pennsylvania State University, Department of Astronomy \& Astrophysics, 525 Davey Lab, \\ University Park, PA 16802, USA \\ email: mrichards@astro.psu.edu
}

\begin{abstract}
Three-dimensional Doppler tomography of interacting binaries has now provided some interesting perspectives of the gas flows beyond the central plane corresponding to the orbital plane. These images suggest that the magnetic field of the mass losing star influences the gas flows in some cases. Earlier 2D tomograms displayed evidence of gas flows associated with the gas stream, accretion disks, accretion annuli, and hot spots as well as evidence of magnetic flows associated with the mass loser. These indirect images have revealed the active environments that exist in the slow-mass-transfer Algols, cataclysmic variables, polars, x-ray binaries, and gamma ray binaries.
\end{abstract}

Keywords. accretion, accretion disks, (stars:) binaries (including multiple): close, (stars:) binaries: eclipsing, (stars:) binaries: general, (stars:) circumstellar matter, stars: imaging, techniques: image processing, X-rays: binaries, gamma rays: observations, stars: individual $(\beta$ Per, $\mathrm{U}$ CrB, RS Vul, V711 Tau, TT Hya, AU Mon, Cyg X-1, o Ceti)

\section{Introduction}

We can resolve images of distant galaxies and yet most nearby stars remain unresolved. We have high resolution images of the Sun in 2D and 3D, but not for other stars. In the case of interacting binaries, it is challenging to resolve the images of the stars since the angular separations are in the milli-arcsecond regime, with separations comparable to the Mercury-Sun distance. However, spectra provide us with information about the Doppler motions of the gas between and around the stars. Moreover, most interacting binaries contain a magnetically active star like our Sun, and recent images of the Sun from the Solar Dynamics Observatory demonstrate the immense level of activity associated with the Sun; yet we often treat cool stars in binaries as dormant objects when their magnetic activity is more powerful than that of the Sun. The magnetically-controlled structures on the cool sun-like mass-losing star (e.g., prominences) could influence how the gas flows between the stars in a binary.

Well-resolved images of single stars have been achieved in only a few cases, e.g., Betelgeuse, Sirius, Altair, Vega (Haubois et al. 2009). However, the CHARA and NOI interferometers have now produced the first resolved optical images of the brightest and nearest eclipsing binary and triple-star system in the sky: Algol, $\beta$ Per (Czismadia et al. 2009; Zavala et al. 2010). The Navy Optical and Infrared Interferometer (NOI) is composed of six $1.8 \mathrm{~m}$ mirrors with a resolution of 0.2 milli-arcsecond resolution while the Center for High Angular Resolution Astronomy Interferometer (CHARA) is composed of six 1m mirrors with a resolution of 0.15 milli-arcsecond resolution. At radio wavelengths, Peterson et al. (2010) used a global very long baseline interferometer array to find evidence of asymmetric magnetic structures that are not confined to the orbital plane in a resolved $15 \mathrm{GHz}$ radio image of $\beta$ Per. 
Hydrodynamic simulations have been used to illustrate how the gas should be distributed between stars (e.g., in Algol binaries: Blondin, Richards \& Malinkowski 1995; Richards \& Ratliff 1998). However, direct images showing the flow of gas between stars in an interacting binary were first obtained by Karovska et al. (2005) from soft X-ray Chandra and HST images of a system called Mira (o Ceti). In all other cases, we have to resort to indirect techniques to study the emission sources and gas flows in the binary. Tomography has been used effectively to generate indirect images of these gas flows, and it will be beneficial until interferometers can provide detailed direct images.

\section{Tomography}

The image reconstruction technique of tomography has been used successfully in many fields, including medicine, geophysics, archaeology, oceanography, and astronomy. The basic procedure can be implemented in two steps: (1) acquire a set of views of the object at directions around the object over the $360^{\circ}$ range of angles. These views are called slices or projections that can be represented mathematically by the Radon transform (Radon 1917). (2) The 3D image is recovered through a summation process called back projection; accomplished by taking each image projection and returning it along the path from which it was acquired. The quality of the reconstruction depends on the number of views acquired and the angular distribution of these views. In general, tomography uses (n-1)-dimensional projections to calculate an n-dimensional image of an object (e.g., make a 3D image using 2D projections, like CAT scans in medicine).

The mathematical formulations for 2D and 3D tomography are summarized in Agafonov, Sharova \& Richards (2009). In 2D Doppler tomography, the Radon transform of a function, $I=f\left(v_{x}, v_{y}\right)$, is a set of $1 \mathrm{D}$ projections, $p\left(v_{r}, \phi\right)$, which is the line profile with Doppler shift, $v_{r}$, at each orbital phase, $\phi$, after correction for the systemic velocity.

$$
f\left(v_{x}, v_{y}\right)=\int_{0}^{2 \pi} \int_{-\infty}^{\infty} \int_{-\infty}^{\infty} p\left(v_{r}, \phi\right)|\omega| e^{2 \pi i \omega\left(-v_{x} \cos \phi+v_{y} \sin \phi-v_{r}\right)} d v_{r} d \omega d \phi
$$

This filtered back-projection formula can be extended to the next dimension to calculate $f\left(v_{x}, v_{y}, v_{z}\right)$ (see Appendix of Marsh \& Horne 1988).

The extension to $3 \mathrm{D}$ has been achieved with a technique that is familiar to radio astronomers (e.g., Bracewell \& Riddle 1967). The Radioastronomical Approach (RA) developed by Agafonov \& Sharova (2005) solves the convolution equation $g(x, y, z)=$ $f(x, y, z) * * * h(x, y, z)+n(x, y, z)$, where $g(x, y, z)$ is the summarized image or "dirty map," $f(x, y, z)$ is the brightness distribution of the unknown object, $h(x, y, z)$ is the summarized point spread function, and $n(x, y, z)$ is the noise. This technique can be used for any kind of 3D reconstruction: Cartesian or Doppler. A comparison of the FBP technique with the RA method shows that the RA method produces better results than FBP and with fewer projections (Agafonov, Richards \& Sharova 2006).

The basic assumptions and constraints of Doppler tomography were summarized by Richards, Sharova \& Agafonov (2010). The main assumption is that the line profiles are broadened only by Doppler motions; which is good to first approximation, although turbulent motions may also contribute. We need numerous spectra distributed around the orbit of the binary, collected at high wavelength resolution and with good coverage in orbital phase. The technique has been applied to spectra that are dominated by emission, but the generality of the equations suggests that the method can be applied with caution to absorption spectra as well. In the case of Algol binaries with weak emission-line spectra, we assume that the circumstellar gas is optically thin; which is good to first order. 
The relative gas opacity can be estimated from a comparison between images derived from observed spectra and then from difference spectra. Here the difference spectra are calculated by subtracting the composite spectrum of the stars from the observed spectrum (e.g., Richards 1993). When the RA method is used to derive 3D images, the stretching of the images in the $V_{z}$ direction relative to $V_{x}$ and $V_{y}$ depends on orbital inclination, $i$. The resolutions are the same if $i=45^{\circ}$, however the resolution in $V_{z}$ degrades if $i>45^{\circ}$ up to $90^{\circ}$ (Richards, Sharova \& Agafonov 2010). The stretching effect in the $V_{z}$ direction is linked to the effect of orbital inclination on the Point Spread Function (PSF). Finally, the transformation of the emission intensity from velocity space to Cartesian space is difficult because we first need to know the velocity fields of all emission sources to make the conversion. These velocity fields can be estimated using hydrodynamic simulations.

\section{Application to Interacting Binary Star Systems}

Tomography can be applied only if there exist different angular views of a system. A notable reconstruction of the surface of Venus in Cartesian coordinates was achieved with the use of radar data since the surface is obscured by thick clouds; the reconstruction was achieved with data collected by the Magellan satellite which orbited the planet for four years. However, applications to eclipsing binaries and rotating stars (which provide us with changing views of the system) require the use of spectra, and the technique is termed "Doppler tomography" since the gas motions detected through Doppler shifts are used to provide an image of the gas flows in velocity coordinates (e.g., Marsh \& Horne 1988).

The structures identified from optical and ultraviolet spectra of interacting binaries include classical and transient accretion disks, the gas stream, emission from the chromosphere and other magnetic structures on the donor star (e.g., prominences), shock regions, an accretion annulus, and an absorption zone (where hotter gas is located in the optical tomograms). Accretion disks are found in binaries that contain white dwarfs and neutron stars (e.g., cataclysmic variables, nova-like systems, soft X-ray binaries, gammaray binaries), as well as non-compact main sequence stars (e.g., Algol binaries). The main differences between these systems are: (1) the donor star (compact vs. non-compact) influences the resulting accretion structures; (2) the accretion structures in the compact systems are bright relative to the stars, while the structures in the Algols are faint relative to the luminous main sequence primary star; (3) the large size of the mass gainer in the Algols leads to the direct impact of the gas stream onto the stellar surface in the short-period Algols, while this type of impact does not occur in the long-period Algols or the compact systems. The result is that a complex set of accretion structures is formed in the short-period Algols compared to the classical accretion disks in the other systems.

\section{Indirect 2D and 3D Images of Interacting Binaries}

Numerous time-resolved spectra of the target systems are required for the tomography calculation. Most of the lines are in the optical regime (e.g., for CVs and X-ray binaries: He I $\lambda 5015$, He II $\lambda 4686, \mathrm{H} \beta$; and for Algols and gamma-ray binaries: $\mathrm{H} \alpha, \mathrm{H} \beta, \mathrm{He} \mathrm{I}$ $\lambda 6678$, Si II $\lambda 6371)$. Ultraviolet tomograms were based on the Si IV $\lambda 1063,1073$ doublet and Si IV $\lambda 1394$ line. Doppler tomograms of over 33 CVs have been produced (e.g., Marsh 2001; Morales-Rueda 2004; Steeghs 2004; Schwope et al. 2004). Other images include Xray binaries (e.g., Vrtilek et al. (2004) for SMC X-1 and Her X-1); the nova-like binary V3885 Sgr (Pinja et al. 2012); and the gamma-ray binary LS I+61 303 (McSwain \& 
Richards 2011). The first 3D image of a black-hole candidate Cyg X-1 was made by Sharova et al. (2012).

The first tomograms of systems with non-compact stars (e.g., the Algols) appeared in 1995. Richards (2004) provided a summary of the 2D images of 20 Algols and V711, a detached RS CVn system used as a template to interpret the tomogram of the cool donor star. In 2006, Agafonov, Richards \& Sharova (2006) created the first 3D tomograms for the entire class of interacting binaries using the U CrB alternating Algol system, which displays disk-like emission in the tomogram at some epochs and stream-like emission at other epochs. The 3D tomograms of the disk and stream states of this binary were described in Agafonov, Sharova \& Richards (2009), and compared to RS Vul by Richards, Sharova \& Agafonov (2010).

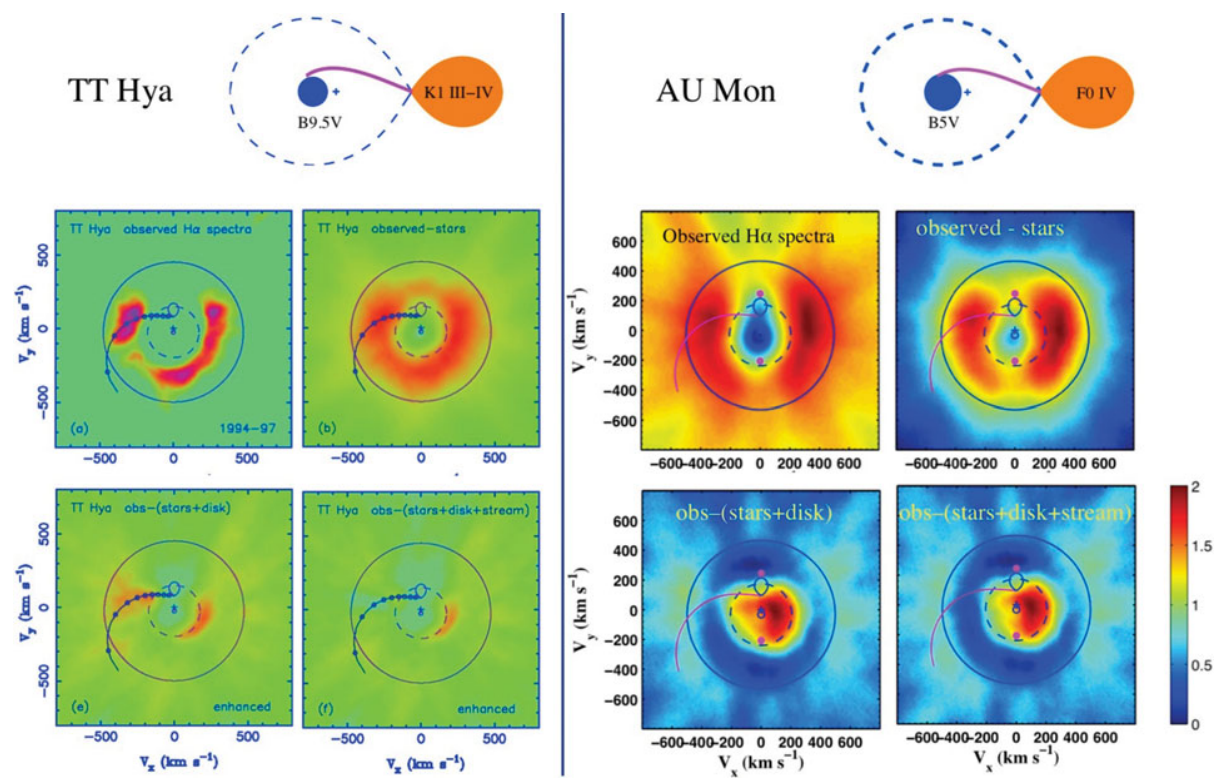

Figure 1. 2D Tomograms of the accretion disk systems TT Hya and AU Mon. The images are based on observed spectra (top left), and when various accretion structures are subtracted from the observed spectra: (1) stellar spectra removed (top right), (2) stars and accretion disk removed (bottom left), and (3) stars, disk and gas stream removed (bottom right). The SHELLSPEC code is effective in isolating the separate parts of the accretion structure, namely accretion disk and gas stream (left frame: Miller et al. 2007; right frame: Atwood-Stone et al. 2011).

\section{Using Synthetic Spectra to Isolate the Sources of Emission}

An innovative technique was developed to isolate the various accretion sources with the help of a spectrum synthesis code called SHELLSPEC (Budaj \& Richards 2004; Budaj, Richards \& Miller 2005). This code was used to model the spectra of the accretion disk and gas stream in the case of TTHya, and 2D tomography was applied to demonstrate how the separate contributors to the tomogram can be isolated (Miller et al. 2007). The results are illustrated in Figure 1. The left-over emission in the TT Hya image (bottom right) represents the asymmetric portion of the disk that was not included in the synthetic spectra; hence the disk in TT Hya is asymmetric. The left-over emission in the AU Mon image represents gas moving at velocities below the Keplerian value. 


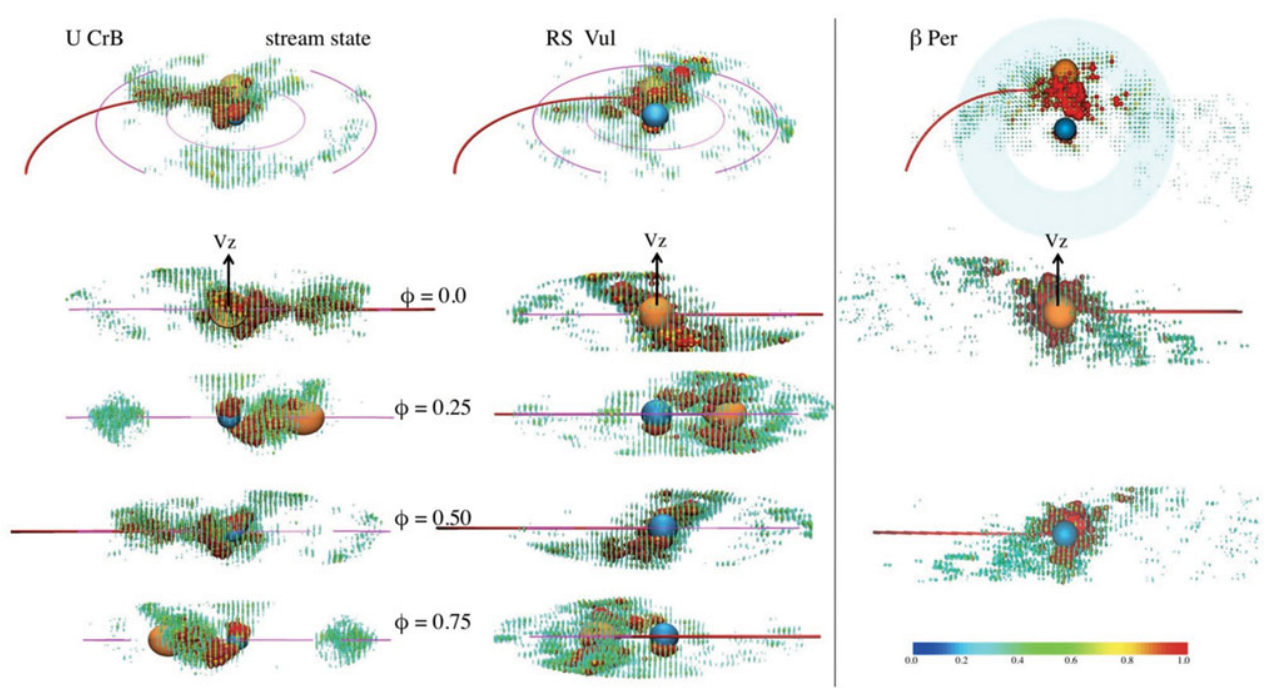

Figure 2. 3D tomograms of U CrB, RS Vul, and $\beta$ Per. The top frames show a tilted view of the velocity distribution of the gas beyond the central plane of each binary, relative to the $V_{z}$ axis. The other views are shown in order of increasing orbital phase, $\phi$. The brightest emission sources shown are the large red points and the fainter sources are small green points (left frame: Richards, Sharova, \& Agafonov 2010; right frame: Agafonov, Sharova, \& Richards 2011).

\section{Summary of Results from 2D and 3D Tomography}

Tomography has been used to make indirect images of interacting binaries at a time when it is not possible to resolve the accretion structures in these systems by any other means. These images provide us with a glimpse of the fascinating structures that form as a result of Roche lobe overflow from a magnetically active star onto a variety of objects from normal stars to compact stellar remnants. We hope to see the direct images of these systems firsthand, perhaps within the next ten years.

The technique has provided new information about interacting binary star systems. These images illustrate the distribution of gas flows in the orbital plane (2D images) and beyond that plane (3D images), and demonstrate the range and complexity of the emission sources in these binaries: from accretion disks and gas streams, to shock regions and magnetic structures. Specific results from the 2D images include evidence of (1) accretion disks around the mass gaining star in the CVs, nova-likes, X-ray binaries, gamma-ray binaries, and long-period Algols; (2) gas streams and gas flowing along magnetic field lines in the magnetic CVs; (3) a combination of Keplerian disks, gas streams flowing along the predicted gravitational path, shock regions where the stream and disk interact, chromospheres, and other magnetic structures in Algol binaries; (4) regions where the gas slows down after circling the mass gainer in direct-impact systems (e.g., Algol, $\beta$ Per); and (5) asymmetric accretion disks that maintain their asymmetry on long timescales (e.g., TT Hya). Additional results from 3D tomography include evidence that (6) prominent and extensive gas flows exist beyond the central plane of the binary in the $\mathrm{z}$ direction (e.g., $\mathrm{U}$ $\mathrm{CrB}$, RS Vul, $\beta$ Per); (7) the accretion disk is tilted or precesses (e.g., U CrB), (7) loop prominences and coronal mass ejections associated with the magnetic field of the donor star also contribute to the gas flows (e.g., RS Vul).

These images have also been used to estimate the gas opacity by comparing the images made from the observed spectra with those made from difference spectra when the stellar 
contribution is removed. The gas was found to be optically thick in the case of CX Dra and optically thin in the case of TT Hya. We can also constrain the properties of the accretion structures (e.g., mass transfer rates, gas densities, gas temperatures) by using synthetic spectra from SHELLSPEC.

\section{Acknowledgements}

This research was supported by NSF grant AST-0908440 and a Distinguished Chairs award from the Slovak Fulbright Commission and the Council for International Exchange of Scholars (CIES). The Visualization Toolkit (VTK) was used in this work.

\section{References}

Agafonov, M. I. \& Sharova, O. I. 2005, AN, 326, 143

Agafonov, M., Richards, M. T., \& Sharova, O. 2006, ApJ, 652, 1547

Agafonov, M., Sharova, O., \& Richards, M. T. 2009, ApJ, 690, 1730

Agafonov, M., Sharova, O., \& Richards, M. T. 2011, ApJ, submitted

Atwood-Stone, C., Miller, B., Richards, M., Budaj, J., \& Peters, G. J. 2011, ApJ, submitted

Blondin, J. M., Richards, M. T., \& Malinkowski, M. 1995, ApJ, 445, 939

Bracewell, R. N. \& Riddle, A. C. 1967, ApJ, 150, 427

Budaj, J. \& Richards, M. T., 2004, Contrib. Astron. Observatory Skalnaté Pleso, 34, 167

Budaj, J., Richards, M. T., \& Miller, B. 2005, ApJ, 623, 411

Csizmadia, S., Borkovits, T., Paragi, Z., et al., 2009, ApJ, 705, 436

Haubois, X, Perrin, G. Lacour, S., et al., 2009, A\&A, 508, 923

Karovska, M., Schlegel, E., Hack, W., Raymond, J., \& Wood B. 2005, ApJ (Letters), 623, 137

Marsh, T. R. 2001, Lecture Notes in Physics, 573, 1

Marsh, T. R. \& Horne, K. 1988, MNRAS, 235, 269

McSwain, M. V. \& Richards, M. T. 2011, in preparation

Miller, B., Budaj, J., Richards, M. T., Koubský, P., \& Peters, G. J. 2007, ApJ, 656, 1075

Morales-Rueda, L. 2004, AN, 325, 193

Peterson, W. M., Mutel, R. L., Gudel, M., \& Goss, W. M. 2010, Nature, 463, 207

Radon, J. 1917, Berichte Schsische Akademie der Wissenschaften Leipzig Math. Phys. Kl., 69, 262 (reprinted in 1983: Proceedings of Symposia in Applied Math, 27, 71)

Prinja, R. K., Long, K. S. Long, Richards, M. T., Witherick, D. K., \& Peck, L. W. 2012, MNRAS, 419,3537

Richards, M. T. 1993, ApJ. Suppl., 86, 255

Richards, M. T. 2004, AN, 325, 229

Richards, M. T., Sharova, O., \& Agafonov, M. 2010, ApJ, 720, 996

Richards, M. T. \& Ratliff, M. A. 1998, ApJ, 493, 326

Schwope, A. D., Staude, A., Vogel, J., \& Schwarz, R. 2004, AN, 325, 197

Sharova, O. I., Agafonov, M. I., Karitskaya, E. A., Bochkarev, N. G. Zharikov, S. V., Butenko, G. Z., \& Bondar, A. V. 2012, these proceedings

Steeghs, D. 2004, AN, 325, 185

Vrtilek, S. D., Quaintrell, H., Boroson, B., \& Shields, M. 2004, AN, 325, 209

Zavala, R. T., Hummel, C. A., Boboltz, D. A., Ojha, R. Shaffer, D. B., Tycner, C., Richards, M. T., \& Hutter, D. J. 2010, ApJ (Letters), 715, L49

\section{Discussion}

V. Neustroev: Please explain how the third dimension can be recovered from 2D observations. What kind of info, observations, in addition to velocities and orbital phases, we need to have in order to get the 3rd dimension in Doppler tomography.

M. RichaRDS: We assume a grid of $V_{z}$ velocities beyond the central plane since we have a fixed view of the binary as set by the orbital inclination. 